\title{
Robust Adaptive Filtering Method for SINS/SAR Integrated Navigation System
}

\author{
Shesheng Gao ${ }^{1}$, Yongmin Zhong ${ }^{2}$, Wei $\mathrm{Li}^{1}$ \\ ${ }^{1}$ School of Automatics, Northwestern Polytechnical University, Xian 710072, China \\ ${ }^{2}$ Curtin University of Technology, Perth, Western Australia 4865, Australia
}

\begin{abstract}
This paper presents a new robust adaptive filtering method for SINS/SAR (Strap-down Inertial Navigation System / Synthetic Aperture Radar) integrated navigation system. This method adopts the principle of robust estimation to adaptive filtering of observational data. A robust adaptive filter is developed to adaptively determine the covariance matrix of observation noise, and adaptively adjust the covariance matrix of system state noise according to the adaptive factor constructed based on predicted residuals. Experimental results and comparison analysis demonstrate that the proposed method cannot only effectively resist disturbances due to system state noise and observation noise, but it can also achieve higher accuracy than the adaptive Kalman filtering method.
\end{abstract}

Keywords: Robust adaptive filtering, adaptive factor, integrated navigation system, Synthetic Aperture Radar, and Strapdown Inertial Navigation System.

\section{Introduction}

The precision of dynamic navigation positioning system depends on cognition and control of disturbances due to dynamic carrier and singular observation. The Kalman filter, which is a commonly used filtering method in a dynamic navigation positioning system, uses the statistical characteristics of the system model to determine estimates recursively [1, 2]. It does not only require accurate system state and observation equations, but it also requires the prior knowledge on the statistical characteristics of system state noise and observation noise. However, due to the influence of a complex environment, it is difficult to accurately obtain the statistical characteristics of system noises, leading to an inaccurate factor to the filter [3]. 
In particular, the inaccuracy between the theoretical behaviour of the filter and its actual behaviour may cause the divergence problem [4].

The federated Kalman filter is a method for optimal fusion of multi-sensor data according to the principle of information distribution [5]. It provides the fault-tolerant ability for the whole system to achieve the overall optimum performance. Although this method overcomes the disadvantages of the classical Kalman filter to some extent, it is still based on precise mathematical models, and can only deal with dynamic linear systems. It also requires real-time calculation of the inverse matrices for each locally estimated error variance matrix, without considering the relevance among the filtering outputs of each individual sensor.

The adaptive Kalman filter is one of the promising strategies for dynamically adjusting the filter parameters based on the estimates of the unknown parameters for on-line estimation of signals and noise statistics. The popular types of the adaptive Kalman filter include the innovation-based adaptive estimation approach, and the adaptive fading Kalman filter approach [4]. In the innovation-based adaptive estimation approach [6-9], the noise distribution matrices employed in the Kalman filter are adjusted according to the dynamic change of observations by using fuzzy logic control. However, the establishment of the fuzzy inference rules and selection of the membership function for fuzzy logic control are difficult problems [10]. The adaptive fading Kalman filter [11-15] incorporates suboptimal fading factors as a multiplier to enhance the influence of innovation information for improvement of the tracking capability in high dynamic maneuvering. Although this filter has a simple structure, it achieves the filtering convergence with the trade-off of the filtering precision $[16]$.

In the recent years, the robust adaptive filter has been used in integrated navigation system to control the influences due to both system state models and observation singularities. Yang et al reported a robust adaptive filter by combining the robust maximum-likelihood estimation with the adaptive filtering process to adaptively adjust the weight matrix of predicted parameters according to the difference between system observation and model information $[17,18]$. This filter can be adaptively converted into the classical Kalman filter, adaptive Kalman filter, and Sage filter by modifying the weight matrix and adaptive factor. However, when the observational information is not sufficient at some epochs, the filtering 
method is difficult to estimate state parameters at these epochs. They also developed a robust adaptive filter with multiple adaptive factors [19]. Although the robustness is improved by using multiple adaptive factors, this method causes an extra computational load, as it requires the number of observations at all calculation epochs be larger than the number of state components. Ding et al reported a process noise scaling method to improve the robustness of adaptive filtering [3]. This method monitors the status of the filter operation by using covariance matching. However, it cannot optimally distribute noises to each individual source. In general, robust adaptive filtering is a challenging research problem [20], and thus its application in integrated navigation system is still very limited.

The SINS/SAR (Strap-down Inertial Navigation System / Synthetic Aperture Radar) integrated navigation system obtains the positioning information by using correlated images. The SINS information can be used for motion compensation and stable alignment of antennae to correct the errors of SAR. In reverse, according to the target information, SAR can also correct the errors of SINS, which are increased over time. Due to the complementary nature of INS and SAR, SINS/SAR integrated navigation system provides a promising solution for achieving high-precision positioning navigation. However, the state model of SINS/SAR integrated navigation system has a small process noise and is exponentially unstable. The errors caused by the instability of the system state model are accumulated in the navigation process, decreasing the accuracy of navigation. Further, due to the influences of system instability and environment uncertainty, the statistical characteristics of system state and observation noises may not be precisely described, resulting in an inaccurate factor to SINS/SAR integrated navigation system [21].

This paper presents a new robust adaptive filtering method for SINS/SAR integrated navigation system. This method adopts the principle of robust estimation to SINS/SAR integrated navigation system for adaptively resisting the disturbances due to system state and observation noises. A robust adaptive filter is developed to adaptively determine the covariance matrix of observation noise, and adaptively adjust the covariance matrix of system state noise through an adaptive factor constructed based on predicted residuals. In order to effectively control the influences of observation and model errors on state parameter estimation for improving filtering accuracy, the adaptive factor acts on the equivalent 
covariance matrix of the whole predicted state vector, rather than on the covariance matrix of the model errors or the covariance matrix of the estimated state vector at the previous epoch. Experiments and comparison analysis have been conducted to comprehensively evaluate the performance of the proposed filtering method for INS/SAR integrated navigation system.

\section{Mathematical Model of INS/SAR Integrated Navigation System}

\subsection{System state equation}

SINS is used to establish the inertial navigation model. The base coordinate system for establishment of the kinematic model is the E-N-U geography coordinate system. The continuous state of the SINS/SAR integrated navigation system can be described as

$$
\dot{X}(t)=F(t) X(t)+G(t) W(t)
$$

where $X(t)$ is the system state vector, which includes various kinds of system errors. $F(t)$ is the dynamic matrix of the state transition, $W(t)$ is the system noise, and $G(t)$ is the noise coefficient matrix.

In order to stabilize the altitude of SINS, the barometric altimeter is introduced into the INS/SAR integrated system. Therefore, the system state vector $X(t)$ can be defined as

$$
X(t)=\left[\delta v_{E}, \delta v_{N}, \delta v_{U}, \delta \varphi, \delta \lambda, \delta h, \phi_{E}, \phi_{N}, \phi_{U}, \varepsilon_{x}, \varepsilon_{y}, \varepsilon_{z}, \nabla_{X}, \nabla_{Y}, \nabla_{Z}, \delta h_{b}\right]^{T}
$$

where $\left(\delta v_{E}, \delta v_{N}, \delta v_{U}\right)$ is the velocity error, $(\delta \varphi, \delta \lambda, \delta h)$ is the position error, $\left(\phi_{E}, \phi_{N}, \phi_{U}\right)$ is the attitude error, $\left(\varepsilon_{x}, \varepsilon_{y}, \varepsilon_{z}\right)$ is the gyro constant drift, $\left(\nabla_{X}, \nabla_{Y}, \nabla_{Z}\right)$ is the accelerometer zero-bias, and $\delta h_{b}$ is the altimeter bias.

The system noise $W(t)$ is defined as 


$$
W(t)=\left[w_{\varepsilon x}, w_{\varepsilon y}, w_{\varepsilon z}, w_{\varepsilon m x}, w_{\varepsilon m y}, w_{\varepsilon m z}, w_{\nabla X}, w_{\nabla Y}, w_{\nabla Z}, w_{\Psi x}, w_{\Psi y}, w_{\Psi z}, w_{h b}\right]^{T}
$$

where $\left(w_{\varepsilon x}, w_{z y}, w_{\varepsilon z}\right)$ is the observation error caused by the gyro's constant drift, $\left(w_{\varepsilon m x}, w_{\varepsilon m y}, w_{\varepsilon m z}\right)$ is the observation error caused by the first-order Markov drift, $\left(w_{\nabla X}, w_{\nabla Y}, w_{\nabla Z}\right)$ is the observation error due to the accelerator's zero deviation, $\left(w_{\Psi x}, w_{\Psi y}, w_{\Psi_{z}}\right)$ is the antenna's attitude angle error of SAR, and $w_{h b}$ is the error due to the altimeter bias.

\subsection{System observation equation}

Since both SINS and SAR can output the latitude, longitude and heading angle for the position of a dynamic carrier, the observation of SINS/SAR integrated navigation system can be treated as the subtraction in the latitude, longitude and heading angle between SINS and SAR, i.e.,

$$
Z(t)=\left[\varphi_{I}-\varphi_{S} \quad \lambda_{I}-\lambda_{S} \quad \psi_{I}-\psi_{S}\right]
$$

where $Z(t)$ is the system observation, $\left(\varphi_{I}, \lambda_{I}, \psi_{I}\right)$ is the latitude, longitude and heading angle of SINS, and $\left(\varphi_{S}, \lambda_{S}, \psi_{S}\right)$ is the latitude, longitude and heading angle of SAR.

According to the different significances of the output variables, $Z(t)$ may be rewritten as

$$
Z(t)=\left[\begin{array}{c}
\varphi_{I}-\varphi_{s} \\
\lambda_{I}-\lambda_{s} \\
\psi_{I}-\psi_{s}
\end{array}\right]=\left[\begin{array}{c}
\left(\varphi+\delta \varphi_{I}\right)-\left(\varphi+\delta \varphi_{S}\right) \\
\left(\lambda+\delta \lambda_{I}\right)-\left(\lambda+\delta \lambda_{S}\right) \\
\left(\psi+\delta \psi_{I}\right)-\left(\psi+\delta \psi_{S}\right)
\end{array}\right]=\left[\begin{array}{c}
\delta \varphi_{I} \\
\delta \lambda_{I} \\
\delta \psi_{I}
\end{array}\right]-\left[\begin{array}{c}
\delta \varphi_{S} \\
\delta \lambda_{S} \\
\delta \psi_{S}
\end{array}\right]
$$

where $\left(\delta \varphi_{S}, \delta \lambda_{S}, \delta \psi_{S}\right)$ is the latitudinal error, longitudinal error and heading angle error of 
SAR, which are all assumed as a white noise process, and $\left(\delta \varphi_{I}, \delta \lambda_{I}, \delta \psi_{I}\right)$ is the latitudinal error, longitudinal error and heading angle error of SINS.

Therefore, the observation of the SINS/SAR integrated navigation system can be further described as

$$
Z(t)=A(t) X(t)+e(t)
$$

where $A(t)$ is the observation matrix, and $e(t)=\left[\delta \varphi_{S}, \delta \lambda_{S}, \delta \psi_{S}\right]^{T}$ is the observation white noise of SAR.

\section{Robust Adaptive Filtering for SINS/SAR Integrated Navigation System}

The robust adaptive filter for the SINS/SAR integrated navigation system is designed based on the adaptive Kalman filter by constructing the adaptive factor according to predicted residual statistic.

By applying Taylor series expansion to (1) and (6), the discrete system state equation is

$$
X_{k}=\Phi_{k, k-1} X_{k-1}+W_{k}
$$

where $X_{k-1}$ is the state vector at epoch $t_{k-1}, \Phi_{k, k-1}$ is the discrete state transition matrix, and $W_{k}$ is the error vector of the kinematic model, whose covariance matrix is $\sum_{W_{k}}$. Correspondingly, the discrete predicted state equation may be written as

$$
\bar{X}_{k}=\Phi_{k, k-1} \hat{X}_{k-1}
$$

where $\bar{X}_{k}$ is the predicted state vector at epoch $t_{k}$, and $\hat{X}_{k-1}$ is the estimate of $X_{k-1}$. The observation equation is 


$$
Z_{k}=A_{k} X_{k}+e_{k}
$$

where $Z_{k}$ is the observation vector, $A_{k}$ is the observation matrix, and $e_{k}$ is the observation noise vector, whose mathematical expectation value is zero and covariance matrix is

$$
\sum_{i, j}= \begin{cases}\sum_{k} & i=j \\ 0 & i \neq j\end{cases}
$$

Obviously, $e_{k}$ is a Gaussian noise vector.

Assume that the residual error equation and predicted residual error equation are

$$
V_{k}=A_{k} \hat{X}_{k}-Z_{k}
$$

and

$$
V_{\overline{\mathrm{X}}_{\mathrm{K}}}=\hat{X}_{k}-\bar{X}_{k}
$$

where $V_{k}$ is the residual vector, $A_{k}$ is the observation matrix, and $V_{\overline{\mathrm{X}}_{\mathrm{K}}}$ is the residual vector of predicted state vector $\bar{X}_{k}$. According to (11) and (12), the following conditional extremum may be established

$$
\Omega_{k}=V_{k}^{T} \bar{\Sigma}_{k}^{-1} V_{k}+\alpha_{k} V_{\bar{X}_{k}}^{T} \Sigma_{\bar{X}_{k}}^{-1} V_{\bar{X}_{k}}-2 \lambda_{k}^{T}\left(A_{k} \hat{X}_{k}-Z_{k}-V_{k}\right)=\min
$$

where $\bar{\Sigma}_{k}^{-1}=\bar{P}_{k}$ is the equivalent weight matrix of $Z_{k}, \Sigma_{\bar{X}_{k}}^{-1}=P_{\bar{X}_{k}}$ is the weight matrix of $\bar{X}_{k}, \alpha_{k}\left(0<\alpha_{k} \leq 1\right)$ is the adaptive factor, and $\lambda_{k}$ is the Lagrange multiplier. $\bar{P}_{k}$ is the 
adaptive estimation of weight matrix $P_{k}=\Sigma_{k}^{-1}$, i.e. $\bar{p}_{i i}=p_{i} w_{i}$, where $w_{i}$ is the weight factor. Therefore, $\bar{P}_{k}$ may be written as

$$
\bar{P}_{k}=\left(\begin{array}{cccc}
\bar{p}_{11} & & & \\
& \bar{p}_{22} & & \\
& & \ddots & \\
& & & \bar{p}_{n n}
\end{array}\right)=\left(\begin{array}{llll}
p_{1} w_{1} & & & \\
& p_{2} w_{2} & & \\
& & \ddots & \\
& & & p_{3} w_{3}
\end{array}\right)=P_{k}
$$

By letting the derivatives of (13) with respect to $V_{k}$ and $\hat{X}_{k}$ are zero, we have

$$
\frac{\partial \Omega_{k}}{\partial V_{k}}=2 V_{k}^{T} \bar{P}_{k}+2 \lambda_{k}^{T}=0
$$

and

$$
\frac{\partial \Omega_{k}}{\partial \hat{X}_{k}}=2 V_{k}^{T} \bar{P}_{k} A_{k}+2 \alpha_{k} V_{\bar{X}_{k}}^{T} P_{\bar{X}_{k}}-2 \lambda_{k}^{T} A_{k}=0
$$

By (15) and (16), we have

$$
V_{k}=-\lambda_{k} \bar{\Sigma}_{k}
$$

and

$$
V_{\bar{X}_{k}}=\frac{1}{\alpha_{k}} \sum_{\bar{X}_{k}} A_{k}^{T} \lambda_{k}
$$

where $\bar{\Sigma}_{k}$ is the equivalent covariance matrix of $Z_{k}, \sum_{\bar{X}_{k}}$ is the covariance matrix of 
$\bar{X}_{k}$, and

$$
\lambda_{k}=\bar{P}_{k}\left(Z_{k}-A_{k} \hat{X}_{k}\right)
$$

By (16),

$$
V_{k}^{T} \bar{P}_{k} A_{k}+\alpha_{k} V_{\bar{X}_{k}}^{T} P_{\bar{X}_{k}}-\lambda_{k}^{T} A_{k}=0
$$

Substituting (19) into (20),

$$
2 V_{k}^{T} \bar{P}_{k} A_{k}+\alpha_{k} V_{\bar{X}_{k}}^{T} P_{\bar{X}_{k}}=0
$$

By applying the matrix transpose operation to (21),

$$
2 A_{k}^{T} \bar{P}_{k} V_{k}+\alpha_{k} P_{\bar{X}_{k}}^{T} V_{\bar{X}_{k}}=0
$$

Substituting (11) and (12) into (22),

$$
2 A_{k}^{T} \bar{P}_{k}\left(A \hat{X}_{k}-Z_{k}\right)+\alpha_{k} P_{\bar{X}_{k}}^{T}\left(\hat{X}_{k}-\bar{X}_{k}\right)=0
$$

By solving (23), the following equation may be written

$$
\hat{X}_{k}=\left(A_{k}^{T} \bar{P}_{k} A_{k}+\frac{\alpha_{k}}{2} P_{\bar{X}_{k}}\right)^{-1}\left(A_{k}^{T} \bar{P}_{k} Z_{k}+\frac{\alpha_{k}}{2} P_{\bar{X}_{k}} \bar{X}_{k}\right)
$$

Letting

$$
K_{k}=\left(A_{k}^{T} \bar{P}_{k} A_{k}+\frac{\alpha}{2} P_{\bar{X}_{k}}\right)^{-1} A_{k}^{T} \bar{P}_{k}
$$


and considering

$$
\begin{aligned}
\left(A_{k}^{T} \bar{P}_{k}\right)^{-1} \frac{\alpha}{2} P_{\bar{X}_{k}} \bar{X}_{k} & =\left[\left(A_{k}^{T} \bar{P}_{k}\right)^{-1} \frac{\alpha}{2} P_{\bar{X}_{k}}+A_{k}-A_{k}\right] \bar{X}_{k} \\
& =\left[\left(A_{k}^{T} \bar{P}_{k}\right)^{-1}\left(\frac{\alpha}{2} \bar{P}_{\bar{X}_{K}}+A_{k}^{T} \bar{P}_{k} A_{k}\right)-A_{k}\right] \bar{X}_{k} \\
& =\left(K_{k}^{-1}-A_{k}\right) \bar{X}_{k}
\end{aligned}
$$

(24) may be further written as

$$
\hat{X}_{k}=K_{k}\left[Z_{k}+\left(K_{k}^{-1}-A_{k}\right) \bar{X}_{k}\right.
$$

Substituting (18) and (19) into (11),

$$
\lambda_{k}=\left(\frac{1}{\alpha_{k}} A_{k} \Sigma_{\bar{X}_{k}} A_{k}^{T}+\bar{\Sigma}_{k}\right)^{-1}\left(Z_{k}-A_{k} \bar{X}_{k}\right)
$$

By (27), the solution of the discrete robust adaptive filter can be obtained as

$$
\hat{X}_{k}=\left[I-K_{k} A_{k}\right] \bar{X}_{k}+K_{k} Z_{k}
$$

where $K_{k}$ is the gain matrix, which is represented as

$$
K_{k}=\frac{1}{\alpha_{k}} \Sigma_{\bar{X}_{k}} A_{k}^{T}\left(\frac{1}{\alpha_{k}} A_{k} \bar{\Sigma}_{\bar{X}_{k}} A_{k}^{T}\right)^{-1}
$$


where $\bar{\sum}_{\bar{X}_{k}}$ is the equivalent variance matrix of $\bar{X}_{k}$, which is represented as

$$
\sum_{\bar{X}_{k}}=\frac{1}{\alpha_{k}} \sum_{\bar{X}_{k}}=\frac{1}{\alpha_{k}}\left(\Phi_{k, k-1} \sum_{\hat{X}_{k-1}} \Phi_{k, k-1}^{T}+\sum_{w_{k}}\right)
$$

where $\sum_{w_{k}}$ is the covariance matrix of the input noise vector at $t_{k}$.

$\sum_{\bar{X}_{k}}$ is estimated by the classical Kalman filter at $t_{k}$, i.e.

$$
\sum_{\bar{X}_{k}}=\Phi_{k, k-1} \sum_{\hat{X}_{k-1}} \Phi_{k, k-1}^{T} \sum_{w_{k}}
$$

where $\sum_{\hat{X}_{k-1}}$ is the covariance matrix of $\hat{X}_{k}$ at $t_{k-1}$.

To balance the contributions of observations and predicted states to estimated state parameters, the robust adaptive factor $\alpha_{k}$ is set as a piecewise decreasing function [18]

$$
\alpha_{k}=\left\{\begin{array}{cl}
1 & \left|\Delta \tilde{X}_{K}\right| \leq c_{0} \\
\frac{c_{0}}{\left|\Delta \tilde{X}_{k}\right|}\left(\frac{c_{1}-\left|\Delta \tilde{X}_{k}\right|}{c_{1}-c_{0}}\right)^{2} & c_{0}<\left|\Delta \tilde{X}_{k}\right| \leq c_{1} \\
0 & \left|\Delta \tilde{X}_{k}\right|>c_{1}
\end{array}\right.
$$

where $c_{0}$ is any value within $1 \sim 1.5$, and $c_{1}$ is any value within 3.0 8.0. $\Delta \tilde{X}_{k}$ is represented as

$$
\Delta \tilde{X}_{k}=\left\|\tilde{X}_{k}-\bar{X}_{k}\right\| / \sqrt{\operatorname{tr}\left(\sum_{\bar{X}_{k}}\right)}
$$

where $\tilde{X}_{k}$ is the robust solution for the state vector at the current epoch, and $\operatorname{tr}(\cdot)$ is the trace of a matrix. 
It is not difficult to see that the adaptive factor of the robust adaptive filter acts on the equivalent covariance matrix of the whole predicted state vector, i.e. $\bar{\sum}_{\bar{x}_{k}}$, rather than on the covariance matrix of the model errors, i.e. $\sum_{w_{k}}$, or the covariance matrix of the estimated state vector at the previous epoch, i.e. $\sum_{\hat{x}_{k-1}}$. Therefore, the proposed robust adaptive filter can effectively control the disturbances on state parameter estimation due to singular observations and singular model noises, providing reliable results for navigation.

\section{Computational Analysis and Discussions}

Experiments have been conducted to evaluate the performance of the proposed filtering method for INS/SAR integrated navigation system. In order to analyze the performance of the proposed filtering method under the flight condition of high velocity, acceleration and flexibility, the flight trajectory of the aircraft is selected as Fig. 1, where " $H$ " denotes the height, "L" denotes the longitude, and “ $\gamma$ " denotes the latitude. As shown in Fig. 1, there are different flight states, such as accelerating, climbing and turning, involved in the flying trajectory. The gyro drift is $0.01^{\circ} / \mathrm{h}$, the first-order Markov process drift of the gyro is $0.001^{\circ} / \sqrt{h}$ (the relevant time is $1 h$ ), and the accelerator zero bias is $10^{-4} \mathrm{~g}$. The horizontal positioning accuracy of SAR is $50 \mathrm{~m}$, the accuracy of the heading angle is 10 , and the output period is $5 s$ (Note: the filtering process is updated only with time when there is no output from SAR). The accuracy of the altimeter is $60 \mathrm{~m}$. The initial position error is $50 \mathrm{~m}$, the initial velocity error is $2 \mathrm{~m} / \mathrm{s}$, and the initial angle error of the platform is $50^{\prime}$. The simulation time is 1500 s, and the filtering period is 1 s. The velocity and position errors obtained by the robust adaptive filtering method are shown in Fig. 2 and Fig. 3, respectively. From Fig. 2 and Fig. 3, it can be seen that the proposed filtering method can effectively control the disturbances due to observation and model noises, and provide reliable results for navigation. 


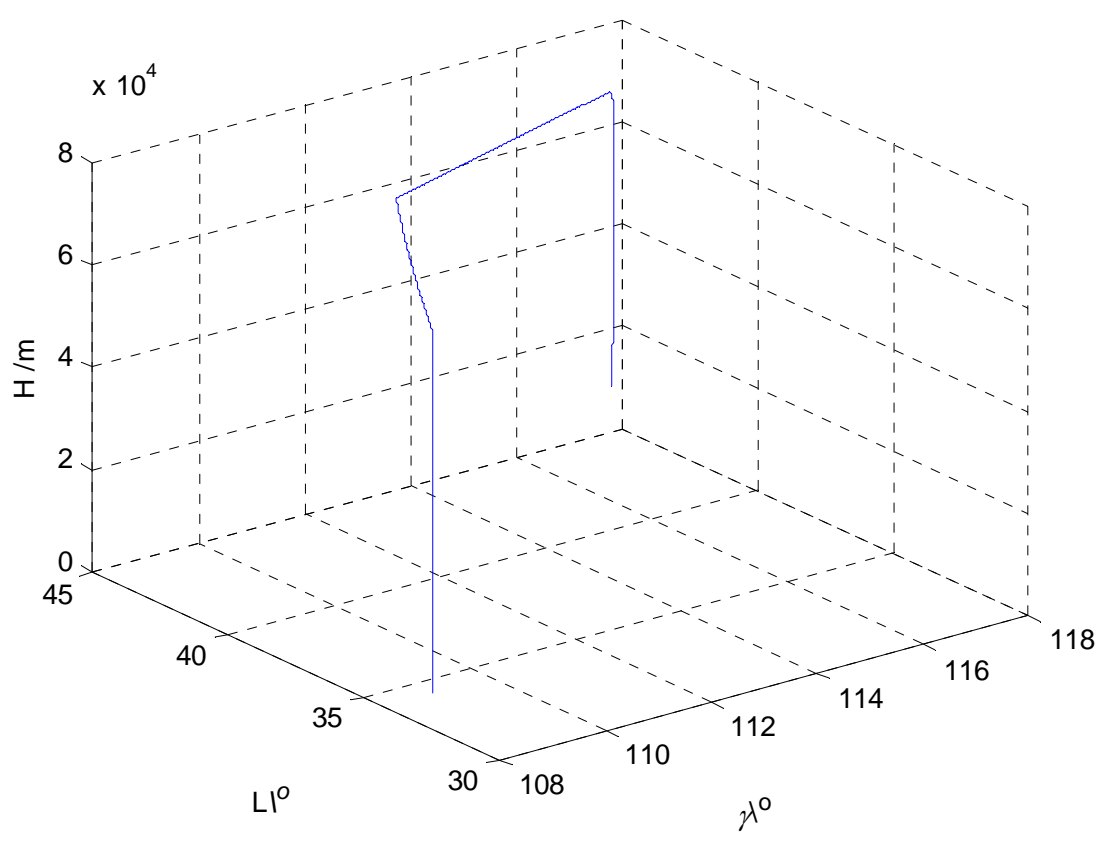

Figure 1. Flight trajectory

The performance of the proposed method has been further evaluated by comparing with the adaptive Kalman filtering method [12]. For the purpose of comparison, experiments have been conducted under the same conditions as Figs. 2 and 3 by the adaptive Kalman filtering method. The velocity and position errors obtained by the adaptive Kalman filtering method are shown in Fig. 4 and Fig. 5, respectively. 

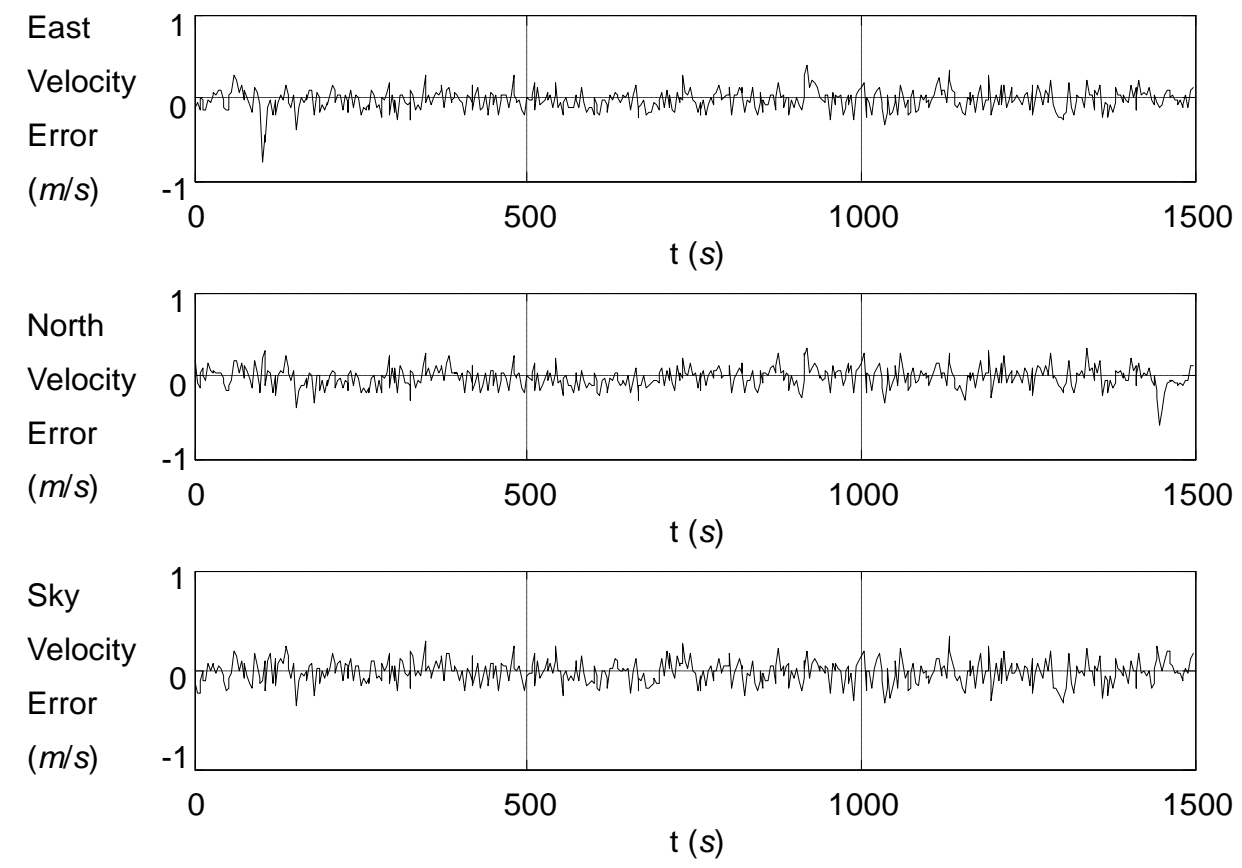

Figure 2. Velocity error obtained by the proposed robust adaptive filtering method
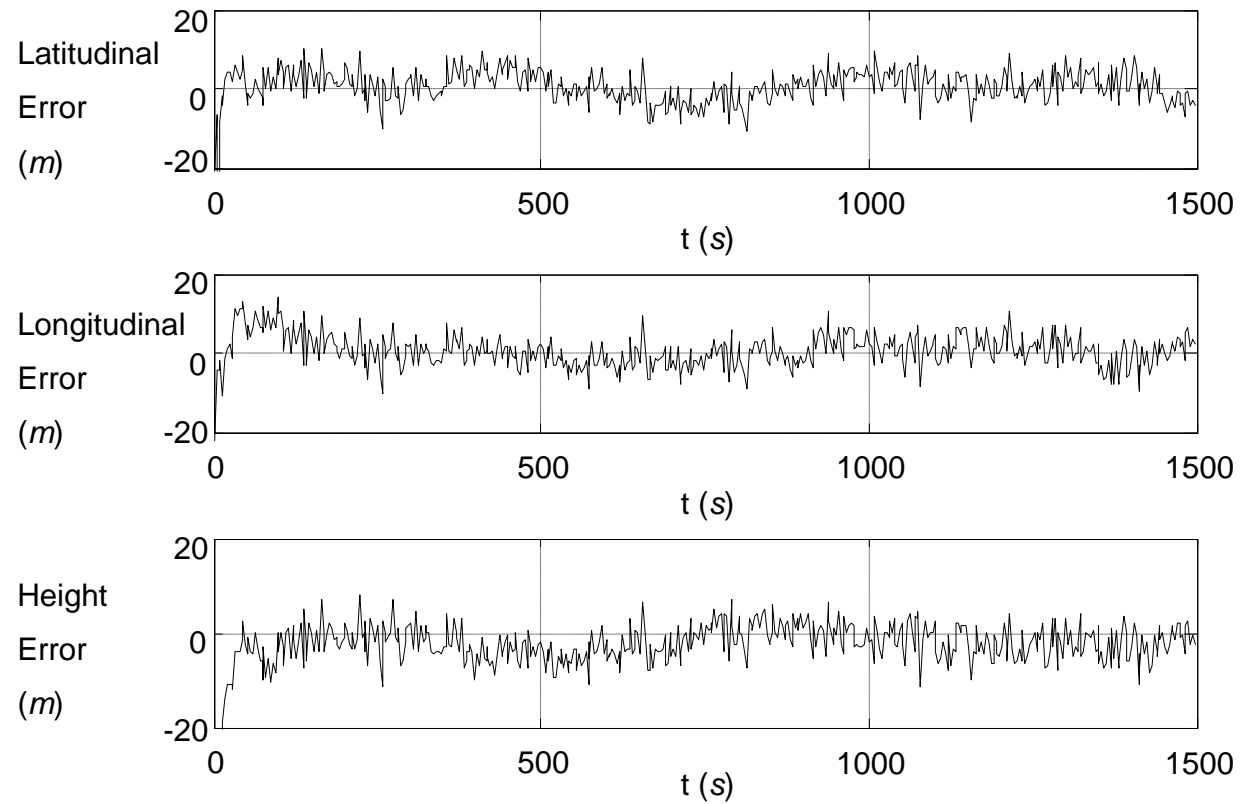

Figure 3. Position error obtained by the proposed robust adaptive filtering method 

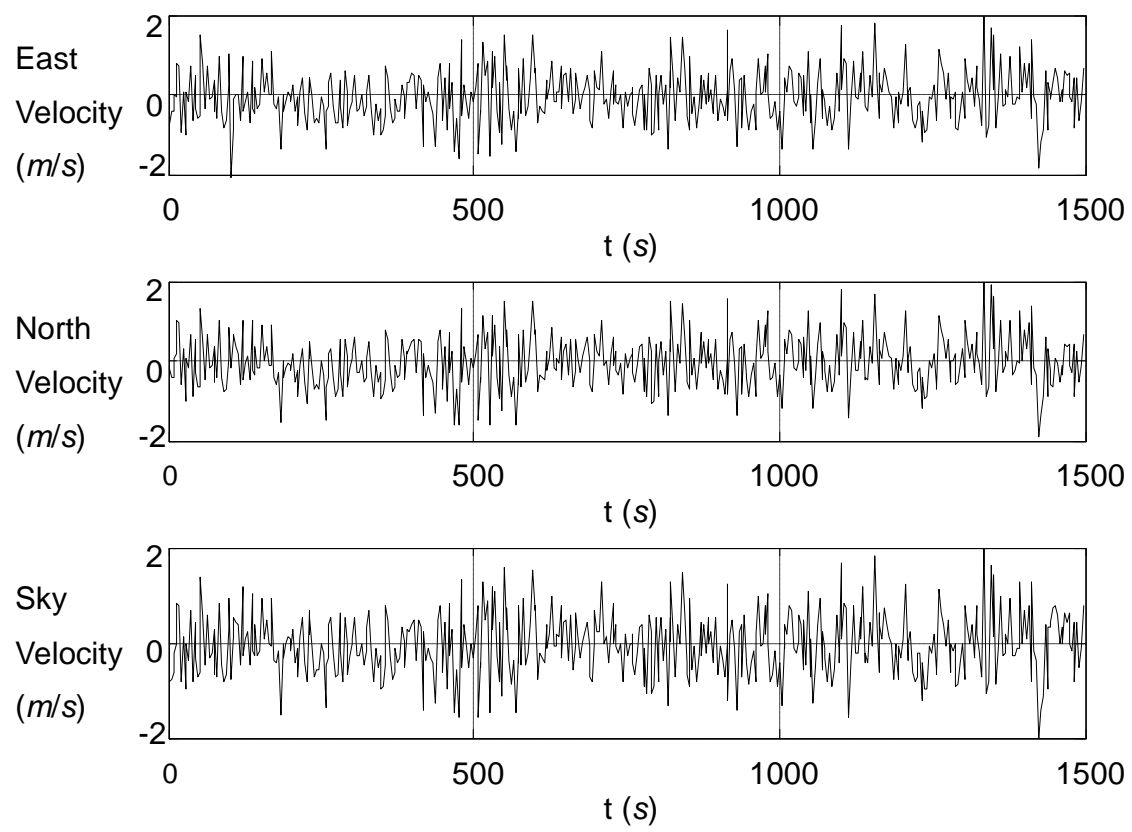

Figure 4. Velocity error obtained by the adaptive Kalman filtering method
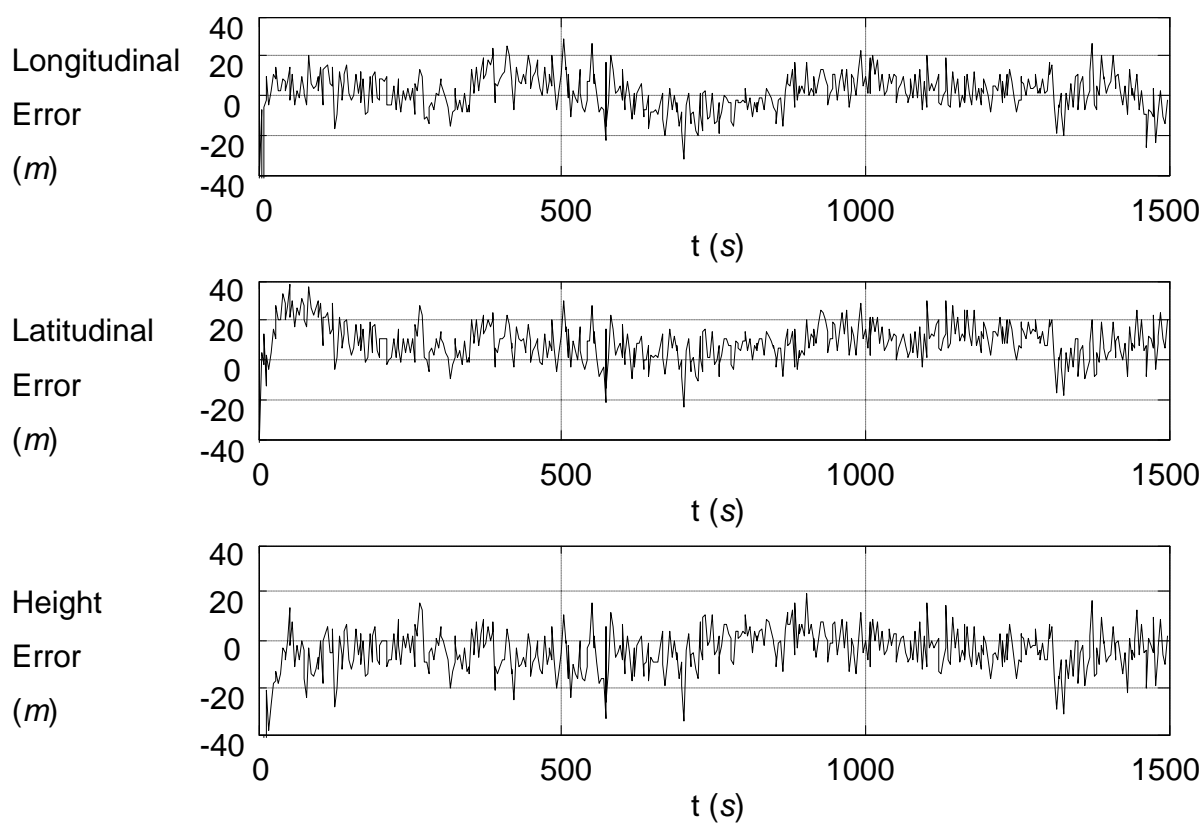

Figure 5. Position error obtained by the adaptive Kalman filtering method

Comparing Fig. 2 with Fig. 4 and Fig. 3 with Fig. 5, it can be seen that the velocity error obtained by the proposed robust adaptive filtering method is within the range of $(-0.5 \mathrm{~m} / \mathrm{s}$, $0.5 \mathrm{~m} / \mathrm{s}$ ), while the velocity error obtained by the adaptive Kalman filtering method is within the range of $(-2 \mathrm{~m} / \mathrm{s}, 2 \mathrm{~m} / \mathrm{s})$. The position error obtained by the proposed robust adaptive 
filtering method is within the range of $(-10 \mathrm{~m}, 10 \mathrm{~m})$, while the position error obtained by the adaptive filtering method is within the range of $(-30 \mathrm{~m}, 30 \mathrm{~m})$. The above experimental results demonstrate that the robust adaptive filtering method can effectively improve the positioning performance of INS/SAR integrated navigation system. The accuracy achieved by the proposed robust adaptive filtering method is significantly higher than that achieved by the adaptive Kalman filtering method. The proposed robust adaptive filtering method is also stable, and can achieve reliable results even in a complex environment.

\section{Conclusions}

This paper presents a new robust adaptive filtering method by adopting the principle of robust estimation to adaptive filtering for INS/SAR integrated navigation system. This method does not only adaptively determine the covariance matrix of observation noise, but it also adaptively adjusts the covariance matrix of system state noise through the adaptive factor. The adaptive factor constructed based on predicted residuals acts on the equivalent covariance matrix of the whole predicted state vector, rather than on the covariance matrix of the model errors or the covariance matrix of the estimated state vector at the previous epoch. The proposed filtering method cannot only control the disturbances on the state estimates due to kinematic model noise and observation noise, but it can also guarantee the reliability of the navigation solution in the cases when observations or predicted values are corrupted. The accuracy of INS/SAR integrated navigation system achieved by the proposed filtering method is also much higher than that by the adaptive Kalman filtering method.

Future work focuses on the improvement of the proposed method by using artificial intelligence. Advanced expert systems and neural networks will be established to intelligent control the filtering process without construction of the adaptive factor for robust filtering the information of INS/SAR integrated navigation system.

\section{Acknowledgements}

The work of this paper is supported by the Aeronautical Science Foundation (China) (Project 
Number: 20080818004) and the Natural Science Foundation of Shaanxi Province (China)

(Project Number: SJ08F04).

\section{References}

1. M. S. Grewal, A. P. Andrews, Kalman Filtering: Theory and Practice using Matlab, John Wiley \& Sons: New York, 2001.

2. A. Gelb, Applied optimal estimation, MIT Press: Cambridge, Massachusetts, 2002.

3. W. Ding, J. Wang, C. Rizos, Improving adaptive Kalman estimation in GPS/INS integration, Journal of Navigation, Vol. 60, No. 3, 2007, pp517-529.

4. D.-J. Jwo, T.-P. Weng, An adaptive sensor fusion method with applications in integrated navigation, The Journal of Navigation, Vol. 61, No. 4, 2008, pp705-721.

5. N. A. Carlson, Federated square root filter for decentralized parallel processes, IEEE Transactions on Aerospace and Electronic Systems, Vol. 26, No. 3, 1990, pp517-525.

6. A. El-Mowafy, A. Mohamed, Attitude determination from GNSS using adaptive Kalman filtering, The Journal of Navigation, Vol. 58, No. 1, 2005, pp135-148.

7. H. Bian, Z. Jin, W. Tian, Study on GPS attitude determination system aided INS using adaptive Kalman filter, Measurement Science and Technology, Vol. 16, No. 10, 2005, pp2072-2079.

8. C. Hide, T. Moore, M. Smith, Adaptive Kalman filtering for low cost INS/GPS, The Journal of Navigation, Vol. 56, No. 1, 2003, pp143-152.

9. A H Mohamed, K. P. Schwarz, Adaptive Kalman Filtering for INS/GPS, Journal of Geodesy, Vol. 73, No. 4, 1999, pp193-203.

10. S. C. Douglas, Introduction to adaptive filters, Digital signal processing fundamentals, V. K. Madisetti (ed.), Taylor \& Francis: Boca Raton, FL, 2010, pp18-1 - 18-18.

11. Y. Geng, J. Wang, adaptive estimation of multiple fading factors in Kalman filter for navigation applications, GPS Solutions, Vol. 12, No. 4, 2008, pp273-279.

12. W. Gao, Y. Yang, X. Cui, S. Zhang, Application of adaptive Kalman filtering algorithm in IMU/GPS integrated navigation system, Geo-Spatial Information Science, Vol. 10, No. 1, 2007, pp22-26.

13. C. Hu, W. Chen, Y. Chen, and D Liu, Adaptive Kalman filtering for vehicle navigation, Journal of Global Positioning Systems, Vol. 2, No. 1, 2003, pp42-47.

14. Q. Xia, M. Rao, Y. Ying, X. Shen, Adaptive fading Kalman filter with an application, Automatica, Vol. 30, No. 8, 1994, pp1333-1338.

15. T. S. Lee, Theory and application of adaptive fading memory Kalman filters, IEEE Transactions on Circuits and Systems, Vol. 35, No. 4, 1988, pp474-477.

16. L. Zhao, X. Wang, J. Ding, Overview of nonlinear filter methods applied in integrated navigation system, Journal of Chinese Inertial Technology, Vol.17, No.1, 2009, pp46-52.

17. Y. Yang, W. Gao, An optimal adaptive Kalman filter, Journal of Geodesy, Vol. 80, No. 4, 2006, pp177-183.

18. Y. Yang, H.-B. He, G. Xu, Adaptively robust filtering for kinematic geodetic positioning, Journal of Geodesy, Vol. 75, No. 2-3, 2001, pp109-116. 
19. Y. Yang, X. Cui, Adaptively robust filter with multi adaptive factors, Survey Review, Vol. 40, No. 309, 2008, pp260-270.

20. A. H. Sayed, M. Rupp, Robust issues in adaptive filtering, Digital signal processing fundamentals, V. K. Madisetti (ed.), Taylor \& Francis: Boca Raton, FL, 2010, pp20-1 20-20.

21. S. Gao, Y. Zhong, X. Zhang, B. Shirinzadeh, Multi-Sensor Optimal Data Fusion for INS/GPS/SAR Integrated Navigation System, Aerospace Science and Technology, Vol. 13, No. 4-5, 2009, pp232-237. 